\title{
A Computational Model of Micro-vascular Growth
}

\author{
Dominik Szczerba and Gábor Székely \\ Computer Vision Lab, ETH, CH-8092 Zürich, Switzerland \\ \{domi, szekely\}@vision.ee.ethz.ch \\ www.vision.ee.ethz.ch
}

\begin{abstract}
In order to supply a growing tissue with oxygen and nutrients and to remove its metabolic wastes, blood vessels penetrating the tissue are formed. Multiple mechanisms are involved in this process ranging over many orders of magnitude: chemical signaling on the bio-molecular level $\left(10^{-9} \mathrm{~m}\right)$, genetic program on the protein level $\left(10^{-7} \mathrm{~m}\right)$, microscopic mechanical cell interactions $\left(10^{-5} \mathrm{~m}\right)$ and external forces and stresses reaching macroscopic scales $\left(>10^{-3} \mathrm{~m}\right.$ ). Better physiological understanding of this phenomenon could result in many useful medical applications, for example in gene therapy or cancer treatment. We present a simulation framework to study mechanical aspects of the micro-vascular growth using techniques from computational geometry, solid mechanics, computational fluid dynamics and data visualization. Vasculogenesis is modeled as traction driven remodeling of an initially uniform tissue in absence of blood flow. Angiogenesis, the subsequent formation and maturation of blood vessels, is handled as a flow driven remodeling of a porous structure resulting from the preceding stage. The mechanical model of tissue response to the traction forces successfully predicts spontaneous formation of primitive capillary networks. Furthermore, we demonstrate that a shear-stress controlled remodeling of such structures can lead to flow fingering effects observed in real cellular media.
\end{abstract}

Keywords: angiogenesis, capillary formation, computational model, microvascular growth, numerical simulation, shear stress, vascular remodeling, vasculogenesis.

\section{Introduction}

Experimental studies confirm that physical forces, including tension, stretching or compression, and flow derived quantities like shear stress influence growth and remodeling in living tissues at the cellular level. In particular, it is known that alignment and structural remodeling of endothelial cells can be modulated by mechano-chemical interactions (e.g. [1], [2] and references therein). It is therefore reasonable to assume that physical interactions between blood flow and endothelial cells play an important role in capillary formation during the vascularization of a growing tissue. For example, [3] demonstrated that flow driven remodelling of transcapillary tissue pillars in the absence of cell proliferation is decisively involved in micro-vascular growth in the chick chorioallantoic membrane. We have recently given a detailed overview of existing mathematical models of vascular growth ([4]). The approaches listed there are generally successful in predicting physiologically realistic systems with relevant biophysical properties, 
but do not attempt to explain the origin of such structures in a microscopic sense. In addition, they either do not address blood flow effects at all, or only implicitly via Poiseuille's Law. In the same paper we have demonstrated that a simple mechanistic model of an explicit tissue response to blood flow suffices to reproduce bifurcation formation and micro-vessel separation from the underlying capillary bed. Structures resulting from the simulations are comparable to real experimental cases (see e.g. [5]) even though the tissue remodeling procedure used in the model is very primitive. The tissue is treated only in a statistical fashion as a set of independent pixels. This allows a very simple treatment of cellular transport but limits possibilities to model the tissue's elastic properties.

In this study we further investigate mechanical aspects of micro-vessel formation but using more realistic physical modeling of the tissue. In contrary to the previous approach, we now treat the tissue as a deformable object and explicitly address its elastic properties. In addition, we now differentiate between the early capillary plexus formation (vasculogenesis) and its subsequent remodeling (angiogenesis). Vasculogenesis is modeled as traction driven reshaping of an initially uniform tissue represented by a mass-spring mesh. In subsequent steps, as a result of fluid-structure interactions and elastic modeling of the tissue's interior, the emerging clusters elongate, align with the flow and merge with their neighbors, which is a prerequisite for the formation of new generations of blood vessels. In order to provide better solution accuracy at the fluidstructure interfaces, where the shear stress is of primary interest, we now also make use of irregular triangular grids. Such meshes can accurately map complex domains but require more advanced formulation of the flow equations than in case of an isometric Cartesian grid. Moreover, this implies additional difficulties in case of dynamically changing boundaries. Due to permanent modifications of the boundary conditions an efficient re-meshing is necessary for the required adaptation of the domain.

In the following section we will explain how we model the elastic response of the tissue and show that it suffices to explain formation of primitive capillary networks. In the next section the geometrical domain will be defined and the corresponding mesh generation technique will be described. Afterwards we will show how we remodel the system using fluid-structure interactions and demonstrate how this leads to the elongation and alignment of the tissue clusters. Finally, in the concluding section we summarize the approach, indicate its implications and propose future developments.

\section{Tissue Modeling}

We start by assuming that living cells consist of a soft matrix (cytoplasm) structurally strengthened by stiff elastic fibers (cytoskeleton). We further assume that a growing tissue increases its volume introducing stretching forces in these fibers, which eventually lead to their spontaneous breaking. At least in the first approximation it is natural to model a system of cytoskeleton fibers as a mesh, where uniformly distributed masses are connected by elastic springs. To describe the behavior of such a mass-spring mesh we start with Newton's equation of motion and assume that the external forces are the sum of linear spring deformation forces (the Hook's Law) and non-conservative inter- 


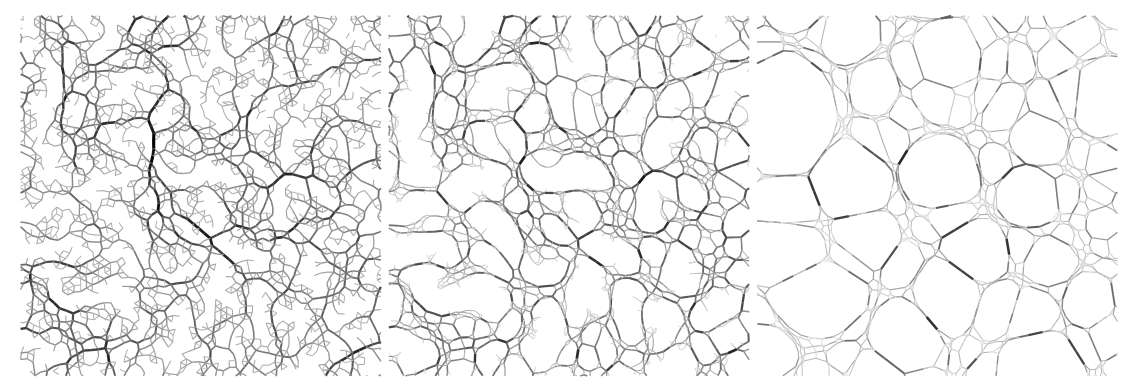

Fig. 1. Computer simulation of a traction-driven remodeling of an initially uniform mesh. The springs are gray- and width- coded according to their stretching forces

nal friction forces due to energy dissipation in cytoskeletal fibers. The resulting motion equation in a matrix form is

$$
M \frac{d^{2} \boldsymbol{r}}{d t^{2}}+D \frac{d \boldsymbol{r}}{d t}+K \boldsymbol{r}+\boldsymbol{F}^{e x t}=0,
$$

where $M$ is the mass matrix, $D$ damping matrix, $K$ stiffness matrix, $\boldsymbol{r}$ is the vector containing the nodal coordinates and $\boldsymbol{F}^{e x t}$ is storing all other external forces (if any). This second order ordinary differential equation is solved numerically using a finite difference scheme. After substituting $d \boldsymbol{r} / d t=\boldsymbol{v}$ and some algebraic rearrangements we obtain the discrete form of the equation, which we use to update the nodal velocities:

$$
\boldsymbol{v}^{n+1}=\frac{1}{1+\frac{D \Delta t}{M}}\left(\boldsymbol{v}^{n}+\frac{\Delta t}{M}\left(\boldsymbol{F}^{K}+\boldsymbol{F}^{e x t}\right)\right),
$$

with $\boldsymbol{v}^{t}$ being a vector of nodal velocities at iteration $t$ and $\boldsymbol{F}^{K}$ the spring deformation forces. Now the nodal positions can be obtained using a simple time integration:

$$
\boldsymbol{r}^{n+1}=\boldsymbol{r}^{n}+\boldsymbol{v}^{n} \Delta t .
$$

A detailed description of the mesh generation procedure will be given in the next section. Here we start from a given uniform unstructured triangular grid covering a unit square. As already mentioned before, we assume that a growing tissue increases its volume and thus stretches its structural fibers. If we start to selectively remove springs that are stretched over a given threshold (damage due to exceeding their mechanical resistance), the initially regular mesh remodels into a set of tissue pillars forming a micro-tubular network, exactly as observed in early stages of vasculogenesis. Fig. 1 shows a few stages of the remodeling process on selected fragments of the whole mesh consisting roughly of 12,000 nodes and 35,000 springs. The springs are width- and gray-coded according to their stretching forces. Our computational model allows to generate results comparable to those presented e.g. in [6] or [7], but as opposed to them, it is not based on a complex mathematical formalism. We do not model cells as scalar fields but base our modeling entirely on physical principles. As we explicitly address the tissue's elastic fibers we do not face numerical difficulties arising from e.g. modeling of surface tension inherent to a density representation. Fig. 2 shows a comparison of one of our remodeling results with a real experimental case found in [7]. 


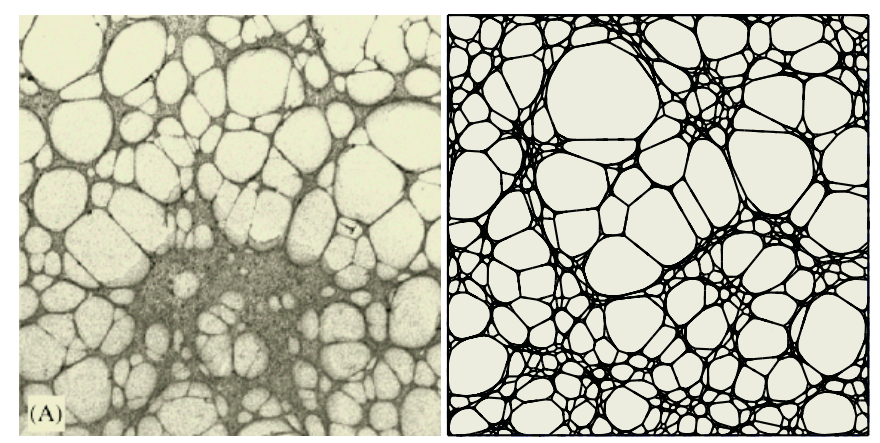

Fig. 2. Left: in vitro angiogenesis experiment from [7] (C) Elsevier, reprinted with permission). Right: computer simulation of a traction-driven remodeling of an initially uniform mesh

\section{Domain Definition and Mesh Generation}

In the previous section we demonstrated how simple mechanistic modeling of cytoskeletal fibers can explain spontaneous formation of micro-channels in an initially homogeneous tissue prior to the actual blood flow. Now we investigate the behavior of the newly created micro-tubules as the blood starts flowing. We note that the resulting network can alternatively be viewed as a set of tissue clusters and the capillaries as narrow passages between them. We now want to approximate the emerging tissue clusters by deformable rings surrounded by an external membrane immersed in the fluid flowing around them. In order to create such rings as well as to generate appropriate meshes necessary to numerically solve the flow equation we need to provide a domain geometry. For that purpose we represent the clusters as polygons, we emit test rays from their centers and run an automatic search for the nearest springs intersecting with them. This information allows us to inspect the topological connections necessary for polygon reconstruction. Unfortunately, the contracting meshes produce many undesired topological artifacts like springs with zero length, degenerated nodal positions or highly non-homogeneous distribution of nodes on the cluster boundary edges. To correctly and still automatically - reconstruct the polygons these special cases have to be differentiated and handled properly. Additional problems arise from very narrow passages that are often formed between pillars. In an extreme case one tubule is represented by only one spring. This introduces additional difficulties into the mesh generation procedure which we address here either by mesh refinement or by merging the two polygons if refinement leads to excessive mesh sizes.

Once the polygons are available we proceed by generating a mesh. We distribute masses inside the domain boundary and outside the interior polygons. Then we create topological connections between the masses using the Delaunay triangulation. Next, we remove all the triangles that lie outside the domain or inside any polygon. The triangles that are left are broken up into a unique set of springs and each spring is assigned a rest length being a desired mesh edge length at this place. When just one rest length is assigned to all the springs, a uniform (isometric) mesh will form. To 
achieve refinement near boundaries one can assign different rest lengths for different springs according to e.g. distances to the nearest boundary or local curvature. Once the rest lengths are assigned, the masses are charged electrically and allowed to evolve. Then re-triangulation is performed to correct for any crossing springs, and the same deformation procedure is applied as described in the preceding section. In addition, the masses that come close enough to the boundary segments are snapped to them and not allowed to detach any more. After some iterations this simple method generated triangular meshes of sufficient quality but the time performance turned out to be very low, mostly because of $N^{2}$ cost of electromagnetic interactions. The performance was significantly improved when magnetic repulsion between the masses was replaced by a slight increase in the desired spring rest lengths to form an "internal pressure" [8].
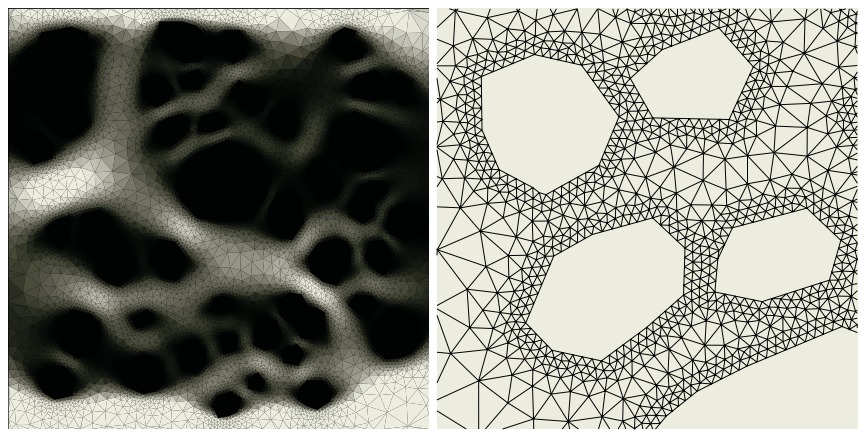

Fig. 3. Left: visualization of velocity in a fragment of a micro-capillary network presented in the previous figures. Right: a fragment of a mesh used to solve the flow equations

\section{Flow Equations and Flow-Tissue Interactions}

To simulate a structural remodeling of a capillary micro-network the key issue is finding the distribution of forces acting in such a physical domain. To calculate flow conditions we use numerical techniques from computational fluid dynamics. The general form of the Navier-Stokes equation for an incompressible Newtonian fluid is

$$
\begin{array}{r}
\rho \frac{\partial \boldsymbol{v}}{\partial t}+\rho(\boldsymbol{v} \cdot \nabla) \boldsymbol{v}=\eta \nabla^{2} \boldsymbol{v}-\nabla p+\boldsymbol{F} \\
\nabla \cdot \boldsymbol{v}=0,
\end{array}
$$

where $\rho$ is the fluid's density, $\eta$ the absolute viscosity, $p$ the pressure, $\boldsymbol{v}$ the velocity and $\boldsymbol{F}$ denotes external forces. Eq.4 represents momentum balance and Eq.5 describes fluid continuity. In the case of creeping flow in capillaries the viscous forces are much stronger than the inertial forces and the convective part of the momentum equation does not need to be included. To describe a steady flow in the absence of external forces the time dependent and body force contributions can also be neglected and the NavierStokes equations can be rewritten as:

$$
\begin{gathered}
\nabla p=\eta \nabla^{2} \boldsymbol{v} \\
\nabla \cdot \boldsymbol{v}=0 .
\end{gathered}
$$


The steady-state discretization results in a system of equations with the form:

$$
\begin{array}{r}
A_{P}^{u} u_{P}+A_{i}^{u} u_{i}=Q_{P}^{u} \\
A_{P}^{v} v_{P}+A_{i}^{v} v_{i}=Q_{P}^{v} \\
a_{i} \boldsymbol{v}_{i} \cdot \hat{n}_{i}=0
\end{array}
$$

with $A$ being the domain dependent coefficient matrix, $Q$ the source terms defined by the boundary conditions, $a$ face surface, $\hat{n}$ face normal, $u, v$ velocity components and using the Einstein summation over direct cell neighbors and the compass notation as in [9]. The formulation used above is the same that we already presented for the finite difference discretization [4], but the coefficients are different and have to be derived separately for the finite volume discretization on unstructured triangular meshes. As these equations have to hold true for every volume element in the mesh, we formulate this as a linear problem $A x=b$, where $x$ is a vector of unknown velocity components and pressure values, $A$ is a domain dependent coefficient matrix and $b$ is defined by the boundary conditions. Unfortunately, Eqs. 8-10 are not suitable for modeling pressure, as pressure is not even present in the continuity equation and is very weakly coupled with the velocity components. Solving such a system of equations in a standard way (i.e. simultaneously) results in oscillatory pressure solutions, therefore this case needs special treatment. To address this problem we adapted the smoothing pressure correction, originally presented for orthogonal grids [10], for the case of unstructured triangular meshes. We continue by solving the partial differential equations on the meshes, generated as described in the previous section (see Fig. 3). This results in velocity and pressure values in the center of each volume element. We assume that eventual evolution of the tissue is governed only by shear-induced traction forces at the boundary, which is well justified from experimental observations. We derive now viscous tractions on the boundary walls using the obtained velocity maps and distribute them onto the original mass-spring mesh nodes. Note, that we use two different meshes, a homogeneous one for the mass-spring deformation and an adaptive one for the discretization of the flow equations. This technique is known as a dual mesh approach. Now the original mesh is allowed to deform determining a new geometry and the whole procedure is repeated in a loop. Blood velocity is visualized by reconstructing nodal velocities from the triangle centers (by solving a linear problem) and using OpenGL to plot triangles with linear color interpolation. Unfortunately, simply letting the pillars hang freely, without addressing their internal structure, does not lead to any realistic remodeling. The pillars are only pushed aside all together, somewhat changing shape, but almost entirely preserving distances between them. Even though preferred paths of flow are clearly visible if the velocity is visualized (see gray-coded velocity plot in Fig. 3), we found no tendencies for the pillars to elongate and merge to form any kind of corridors or future vessel walls. The alignment of the tissue clusters and their interactions with the neighbors, however, are prerequisites for any vessel formation. Before any large scale simulations can take place, a better model of the dynamic behaviour of single tissue clusters has to be found. We assumed that the mechanical model involved in this case, ignoring the internal structure of the pillars, was not sufficient to correctly reproduce the required local phenomena. We concluded that the pillars were not just holes, but deformable membranes filled with elastic tissue - a structure having certain physical properties. 
The springs that we used so far corresponded then to extracellular bindings. Additional springs, much stiffer in order to mimic behavior of solid clusters immersed in a jelly matrix, should now be placed in the pillar interiors, and the whole structure should be separated from the external matrix by a membrane. The structural arrangement of skeletal micro-filaments in cells is not trivial and its proper modeling can be crucial for obtaining correct results. We surround a cell with a membrane that is able to sense shear stress around itself and evaluate its average value. Then we make internal connections between all the membrane's nodes using springs 10 times stiffer than springs in the surrounding matrix. Now, if a part of the membrane senses shear stress higher than average, it will transmit contracting forces to all the internal filaments connected to the activated part. In contrary, if somewhere on the membrane shear stress lower than average is sampled, stretching is induced. Such mechanics successfully predicts elongation of a disk placed in a viscous flow (see Fig. 4). The same procedure applied to a bigger mesh is presented in Fig. 5. As can be seen from the figures, pillars finally elongated, aligned with the flow and subsequently merged with the neighbors, resulting in a chain which is the basic pre-condition for the formation of a future vessel wall. Based on this more realistic model of single cluster dynamics, further investigations on much bigger domains can be performed. If few pillars demonstrate clear tendencies to align and form chains, chances for micro-tubule formation in large scale simulations (involving e.g. thousands of such clusters) become realistic. This will, however, require computer code parallelization and other super-computing techniques due to excessive demand on computational resources that modeling of such domains would require.

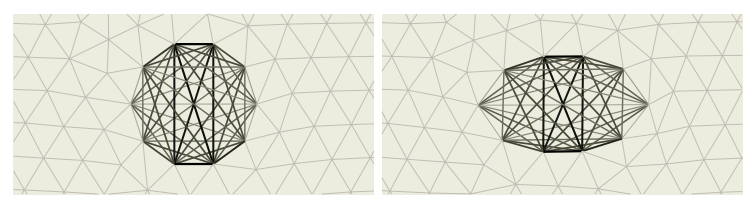

Fig. 4. Deformation of a disk due to its internal mechanics. Flow coming from the left
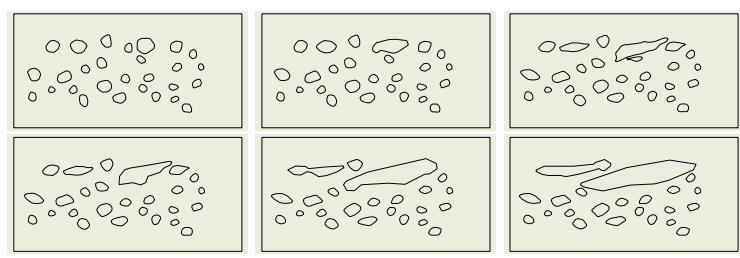

Fig. 5. Elongation of the tissue pillars, alignment with the flow and subsequent merging with neighbors - geometry at different iterations

\section{Conclusions}

We have presented a multi-physics simulation framework to study mechanical aspects of vascular system formation. We use techniques from solid mechanics to model tissue 
response, computational geometry to generate meshes needed to solve partial differential equations, computational fluid dynamics to provide flow conditions in the cellular medium and fluid-structure interactions to simulate tissue remodeling. The simulations, visualized using OpenGL, reveal that mechanical traction forces rebuild an initially homogeneous tissue into a micro-tubular network, as observed in early stages of vasculogenesis. By visualizing blood velocity in such networks we find paths of preferred flow, suggesting future formation of some flow corridors or walls. By addressing the internal structure of the emerging cell clusters we demonstrate their alignment in the flow and tendencies to merge with the neighbors to form chains, which is a prerequisite for future micro-tubule formation. Once a realistic model of single cell clusters is available, large scale simulations, using super-computing due to excessive domain sizes, can be performed in future investigations.

\section{Acknowledgments}

This work is a part of the Swiss National Center of Competence in Research on Computer Aided and Image Guided Medical Interventions (NCCR Co-Me), supported by the Swiss National Science Foundation.

\section{References}

1. Hsiai, T.K., Cho, S.K., Honda, H.M., Hama, S., Navab, M., Demer, L.L., Ho, C.M.: Endothelial cell dynamics under pulsating flows: Significance of high versus low shear stress slew rates. Annals of Biomedical Engineering 30 (2002) 646-656

2. Malek, A.M., Izumo, S.: Mechanism of endothelial cell shape change and cytoskeletal remodeling in response to fluid shear stress. Journal of Cell Science 109 (1996) 713-726

3. Djonov, V., Galli, A., Burri, P.: Intussusceptive arborization contributes to vascular tree formation in the chick chorio-allantoic membrane. Anatomy and Embryology 202 (2000) 347-357

4. Szczerba, D., Székely, G.: Computational model of flow-tissue interactions in intussusceptive angiogenesis. Journal of Theoretical Biology 234 (2005) 87-97

5. Djonov, V., Kurz, H., Burri, P.: Optimality in the developing vascular system: Branching remodeling by means of intussusception as an efficient adaptation mechanism. Developmental Dynamics 224 (2002) 391-402

6. Manoussaki, D.: A mechanochemical model of vasculogenesis and angiogenesis. Mathematical Modelling and Numerical Analysis 37 (2003) 581-599

7. Namy, P., Ohayon, J., Tracqui, P.: Critical conditions for pattern formation and in vitro tubulogenesis driven by cellular traction fields. Journal of Theoretical Biology 227 (2004) 103

8. Persson, P.O., Strang, G.: A simple mesh generator in matlab. SIAM Review, Volume 46 (2), pp. 329-345 (2004)

9. Ferziger, J., Perić, M.: Computational Methods for Fluid Dynamics. Springer-Verlag (2002)

10. Date, A.W.: Complete pressure correction algorithm for solution of incompressible navierstokes equations on a nonstaggered grid. Numerical Heat Transfer, Part B 29 (1996) $441-458$ 